\title{
Visualization of a Fish Behavior Using Successive Three-Dimensional Shape Modeling
}

\author{
Junta Doi, Tetsuo Miyake \\ Junta Doi, Department of Computer Science, Chiba Institute of Technology, Tsudanuma, Narashino, 275-0016 Japan, \\ doi@cs.it-chiba.ac.jp \\ Tetsuo Miyake, Department of Production Systems Engineering, Toyohashi University of Technology, Tempaku, Toyohasi, \\ 440-8580 Japan, miyake@ keisys.tutpse.tut.ac.jp
}

\begin{abstract}
Visualization of a three-dimensional fish behavior based on the successive feature extraction using the time sequential three-dimensional shape modeling is reported. The principle of the modeling is based on the intersected viewing pyramid consisting of each image boundary of the object and the corresponding focal point. Threedirectional video images are simultaneously acquired and sequential solid models are then reconstructed to obtain behavioral parameters such as body shape, skeleton, head, tail, gravity center, direction, attack angle, trajectory, velocity, acceleration, and moving energy. This procedure is entirely automatic requiring no manual operation and appears to have some considerable utility in tracking and measurement of moving objects.
\end{abstract}

Keywords: behavior analysis, fish behavior, multidirectional images, solid modeling

\section{Introduction}

Most of resent behavior analyses are related to the two-dimensional, static/quasi-static or point wise treatment. In this paper, a method is proposed that approximates, reconstructs, and visualizes the three-dimensional shape of the object in respect to the behavioral factors. It is suitable especially for the objects that are deformable, irregular and complicated in shape. This result gives a solid model of a polyhedral expression based on multi-directional and noncontacting picture images. With this modeling successive reconstruction of an object model, from the video images in a frame-to-frame manner, is made with the same coordinate system to show transformation and shape deformation through time (Doi et al., 1987, 1990, 1993). Parameters involved in this analysis are timeto-time varying body shape, skeletal figure, positions of mouth (head), tail and gravity center, directions of body, movement and acceleration, angle of attack, trajectory of movement, velocity, acceleration, and moving energy.

\section{Principle of 3-D Shape Modeling}

The principle of the three-dimensional object shape modeling in this paper is summarized as follows. In the first stage, the silhouette or the external boundary of the object image is extracted from a picture taken with a video camera. The boundary is represented by a polygon as an approximation and is shown by a dashed figure in the viewing planes PLi in Fig. 1. It is represented as a successive chain of vertices. In this system, the degree of approximation is controlled by a curvature parameter limiting the extent to which the curved silhouette line is replaced by a single line segment. In the second stage, a semi-infinite pyramid (view pyramid PMi in Fig. 1) is constructed from a polygonal boundary and the corresponding center of perspective or the lens center of the video camera (Ci in Fig. 1). When multi-directional images are applied, a corresponding number of semiinfinite pyramids are generated in the same way. Then the intersection of all of these multidirectional pyramids or the calculation of the 


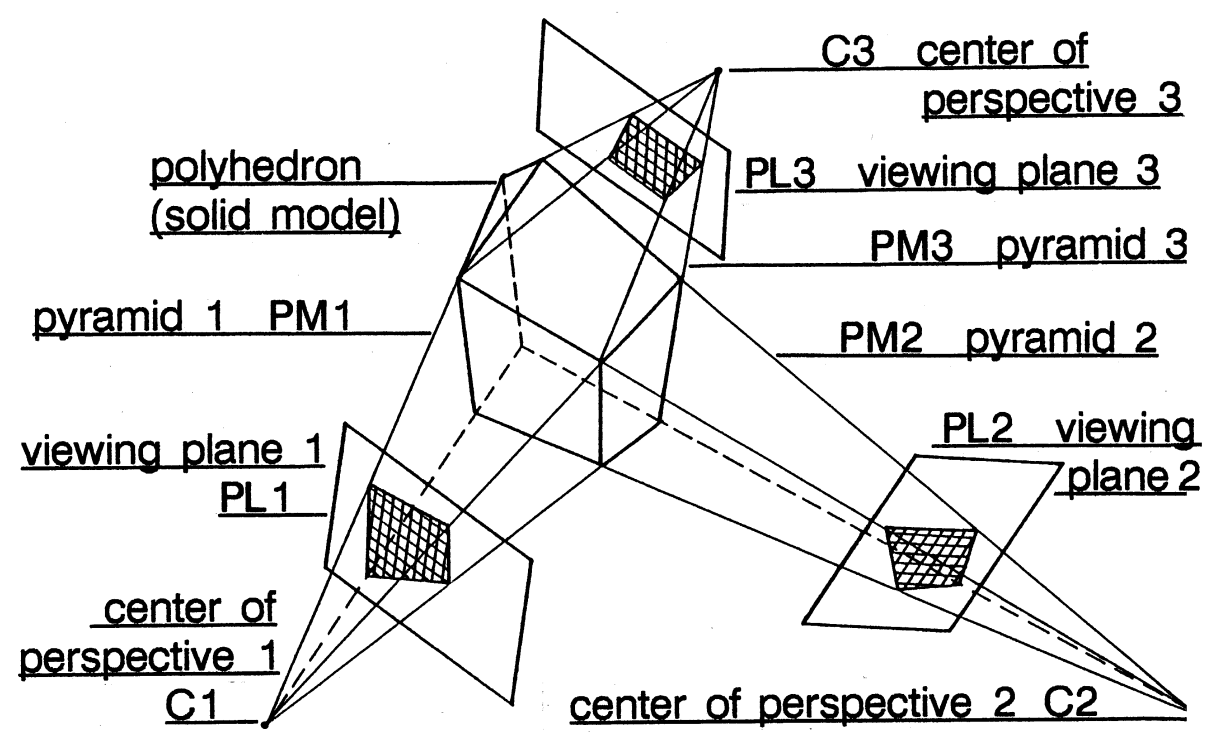

Fig. 1. Principle of the solid modeling.

common region of these pyramids generates a polyhedron.

When the object has a convex shape, this polyhedron becomes a progressively better approximation to the original shape as the number of directions increases. Even if the object is not a thoroughly convex form or the substantial number of visual direction is not large enough, this polyhedron may still often be used as an approximation within acceptable engineering accuracy.

Video camera parameters, such as direction and rotation of the optical axis, field of view, and the magnification ratio are estimated prior to the solid model computation. In this procedure, some calibrating objects such as spheres and rectangular blocks are used.

As shown in the flowchart in Fig. 2, mass properties such as volume, surface, section and gravity center, and features such as shape, axis, endpoints, direction and curvature can be extracted from the reconstructed model. Motion or deformation is obtained by differentiation with respect to time. Behavior is detected from the iteration of this process.

\section{Reconstruction of a Fish Model}

An example of the solid modeling for a plastic fish model, reconstructed from 18-directional video images of $256 \times 240$ picture elements is

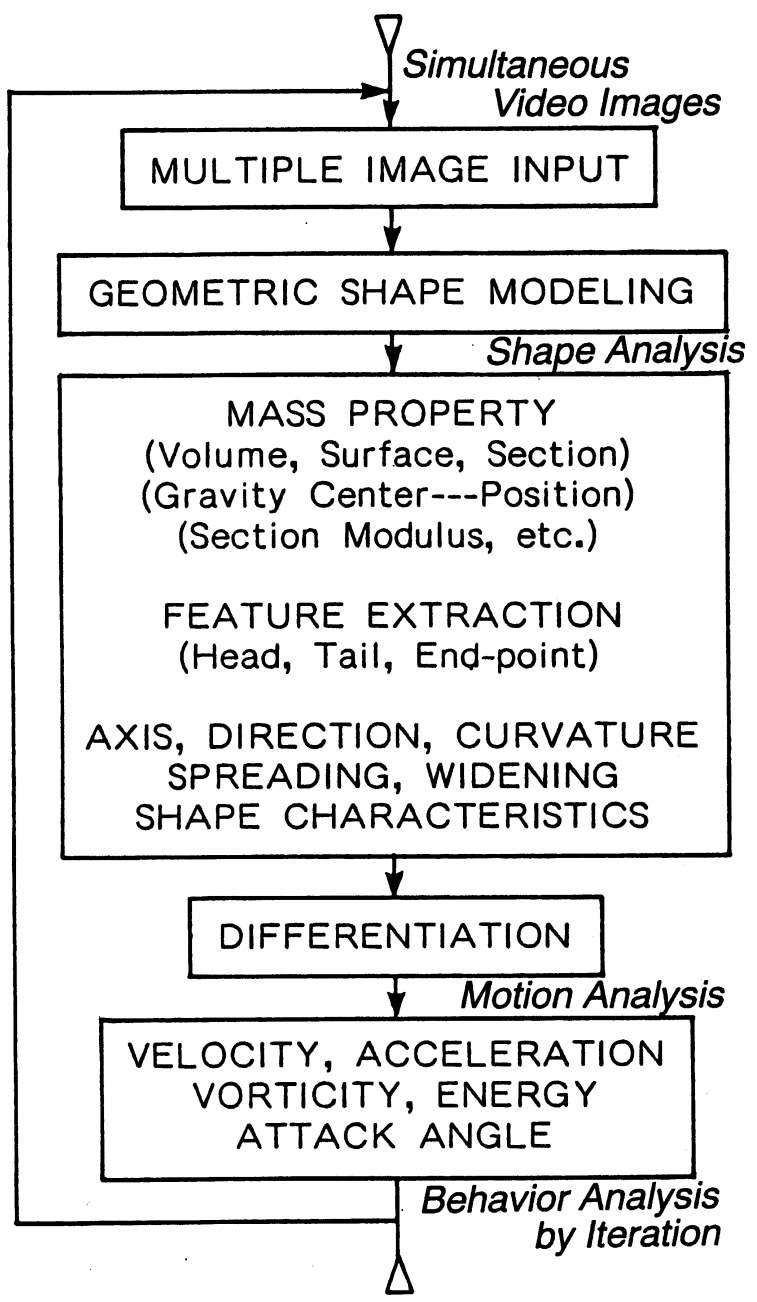

Fig. 2. Flowchart of the analysis. 


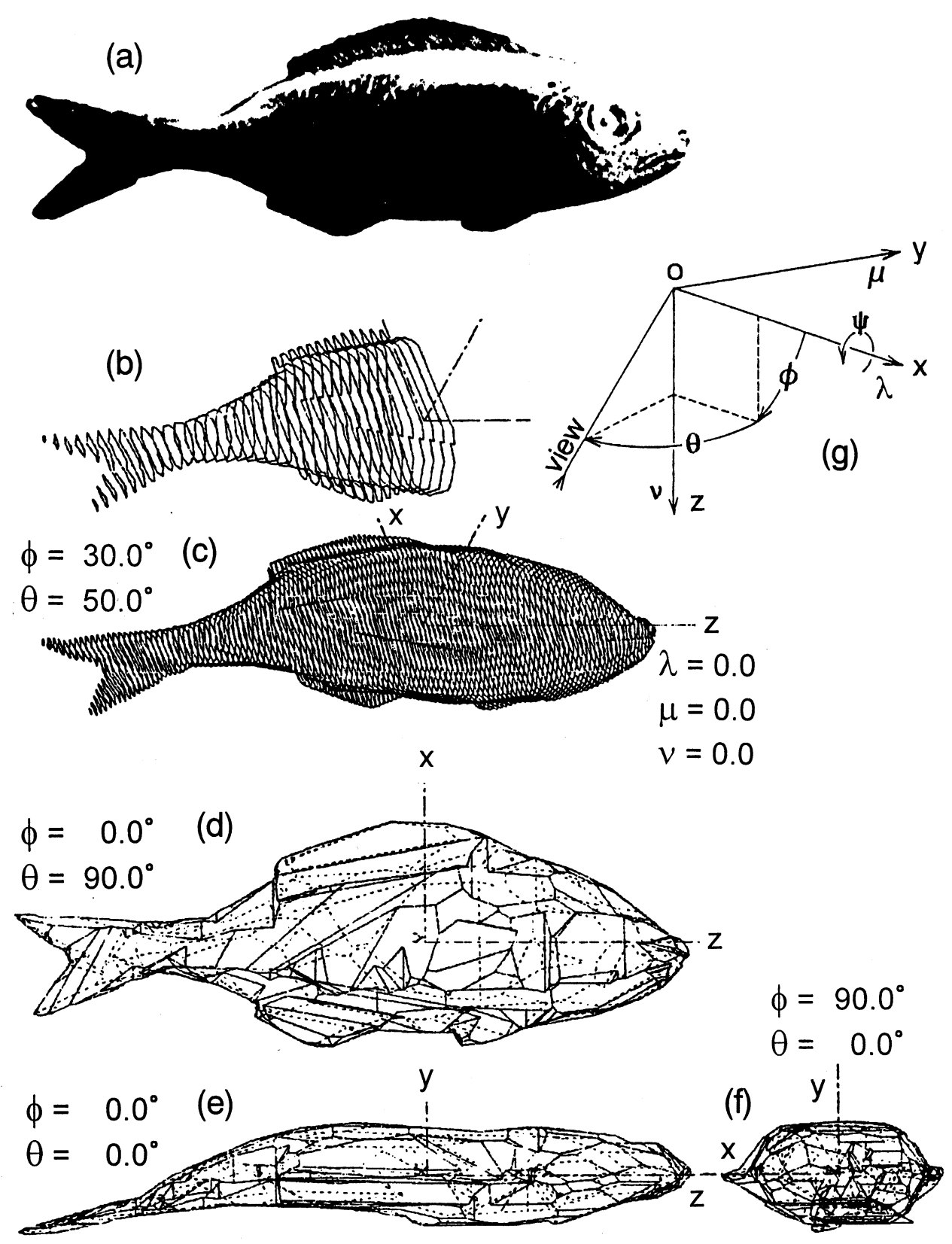

Fig. 3. A 18 directional modeling of a plastic fish.

compared with its photograph in Fig 3(a). The polyhedron consists of 417 facets, 1245 edges and 830 vertices. Figure 3 (c) is a cross sectional representation or contour line display. The section is intersected perpendicular to the skeletal axis at an interval of $1 \mathrm{~mm}$. Moire-fringe-like patterns are observed due to the interference of contour lines on the front and back faces. Figure 3(b) is an example of another partial cross sectional representation at an interval of $2 \mathrm{~mm}$. Figures 3(d), 3(e) and 3(f) are a threedimensional representation by the third angle projection method. Coordinate system, view angle and direction cosine of the cross section are shown in Fig. 3(g). In this test modeling, deviations both in size and volume are usually estimated to be about $0.5 \%$.

\section{Visualization of a Fish Behavior}

A small fish (cyprinodont, medaka), a living test object, was put in a cubic glass vessel of $100 \mathrm{~mm}$ edges. The fish is $33 \mathrm{~mm}$ in total length and is 


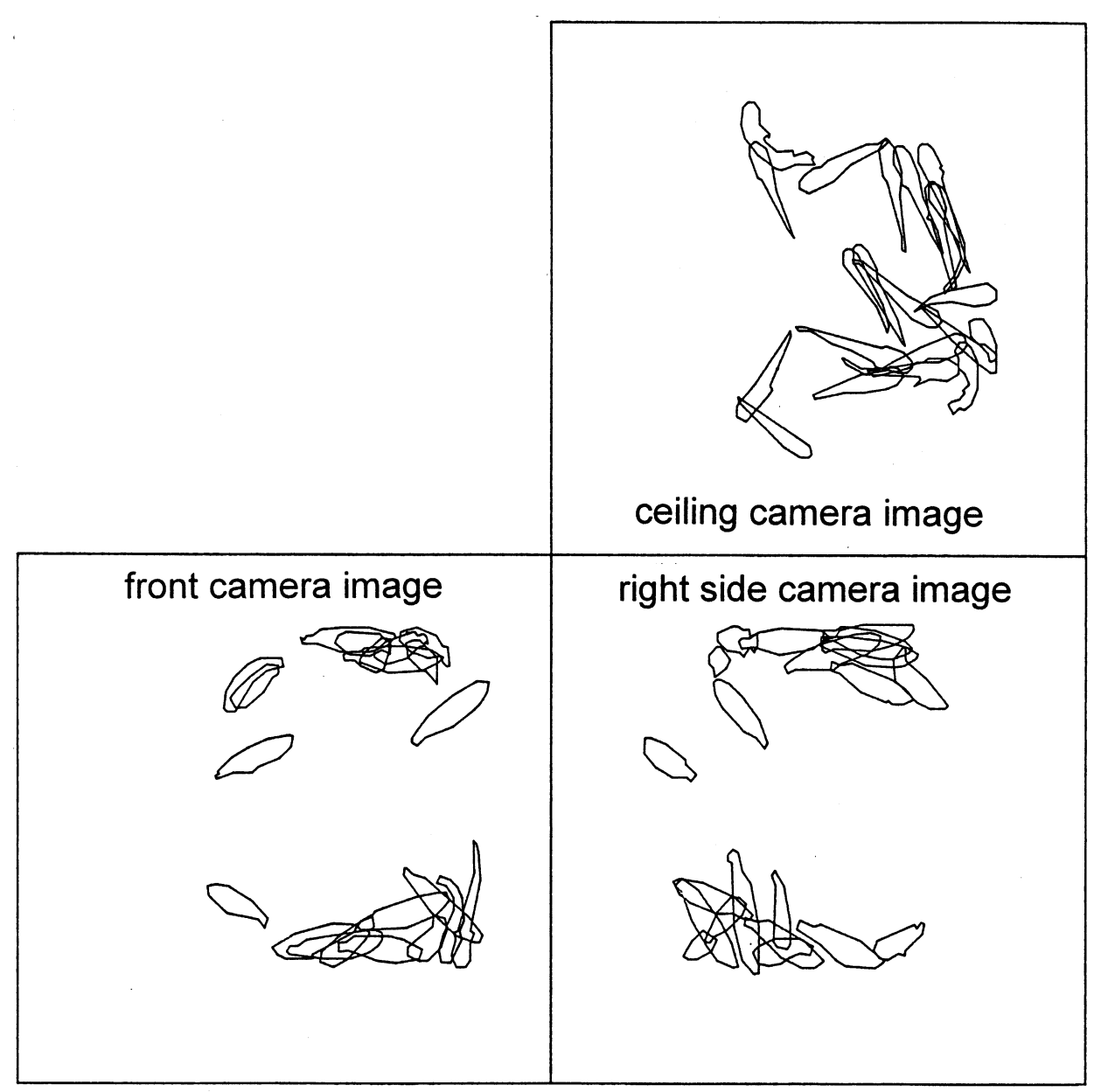

Fig. 4. Three-directional images of the fish.

$26.5 \mathrm{~mm}$ long from the mouth to the end part of the body or to the boundary between the tail and caudal fin, while the fin is $6.5 \mathrm{~mm}$ long. Images of the fish were taken simultaneously by three video cameras installed nearly perpendicular to each other. These three-directional images are shown in Fig. 4. Each fish image is approximated by a polygon with edges of 40 at maximum. All fins, including caudal, dorsal, pelvic and anal fins, are so thin and transparent that none of their images are acquired.

From these three images a polyhedron was constructed as a three-dimensional approximation of the body. Figure 5(a) shows a three-dimensional model of the swimming fish at a moment. Another example of the swimming model, at different moment, is shown in Fig. 5(b) by using the third angle projection method. As this model is made by only three-directional images, the resulting approximation remains rather rough. However, the fundamental shape characteristics are reproduced.
Instantaneous position of the fish is represented by the gravity center of the polyhedron in this study. Skeletal line is approximated by a set of line segment connecting each centroid of the cross section that is cut perpendicular to a longitudinal line of the body at an interval of $1 \mathrm{~mm}$. Here, a skeletal line means a lateral line, similar to the medial axis of the reconstructed polyhedron, not the anatomical spinal column of the real fish. An alternative method to extract more accurate skeletal figure is a socalled skeletonization such as a dedicated thinning procedure (Masuya and Doi, 1995).

The mouth is detected by comparing the distances from the gravity center to both of the end points of the skeletal axis. The closer end point is concluded to be the mouth considering the body shape characteristics of this fish. Accordingly, the opposite endpoint is estimated to be the tail. Positions of the mouth, the gravity center and the tail give the three-dimensional body direction. They also give the angle of attack, 
which is defined as an angle between directions of the body and the movement. Here, the body direction is defined as the tangent of the skeletal line at the gravity center.

Figure 6 is a third angle projection of the fish behavior. The front view in the figure is enlarged separately in Fig. 7. Parameters involved in the figures are positions of the gravity center, mouth (doted) and tail, acceleration at the gravity center, and the trajectory of the movement. The trajectory consists of line segments that sequentially connect every center of gravity in a period of elapsed time. In this paper, each position is calculated in 21 consecutive moments. Time interval is five seconds. Polyhedral models are made using the three simultaneous image data acquired at the same interval. So each line segment between the consecutive gravity cen- ters results in a three-dimensional trajectory of the fish movement in every five seconds. It is equivalent to a mean velocity vector in the same period. Acceleration is calculated from the three-dimensional difference between the two consecutive velocity vectors. In these figures the acceleration vectors are magnified five times for better visibility.

Figure 8 is a simple and intuitive version of Fig. 6. Here, an orthogonal projection from an oblique angle of view is added to the previous third angle projection. This makes threedimensional behavior more comprehensive. A magnified front view is shown in Fig. 9, where a tangential line segment at the gravity center is added. The angle of attack, the angle between the directions of the body and the trajectory,

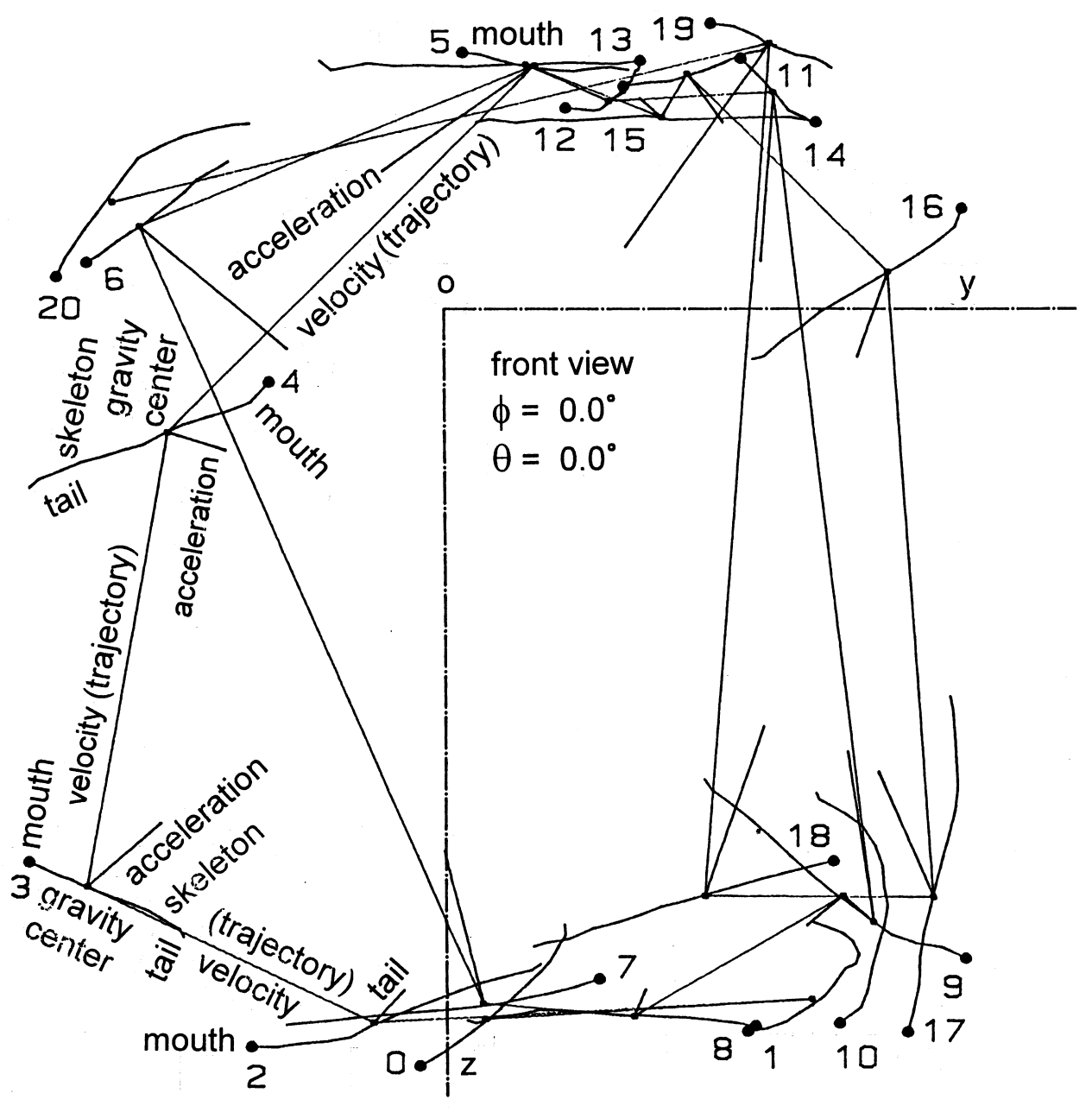

Fig. 7. Front view of the fish behavior. Water surface is around the mouth positions $5,11,13$ and 19 . The bottom is around points $1,2,8,10$ and 17 . 
at every moment becomes intuitive by this presentation. A distinguishing feature of the fish swimming is its large values of the angle. It is due to the mechanism of peculiar propulsion to the fish. A fish can easily change its direction of movement. In the figure large values up to 90 degrees are observed. It is also observed that the fish usually stays near the water surface or the bottom of the vessel. Usually, it faces toward the vertical glass wall and moves on along the wall and seldom crosses diagonally apart from the wall. Leveling cap ability seems not so high, always moving upward or downward.

Distributions of three-dimensional directions of the body, velocity and acceleration are shown in Fig. 10. The top view of the body direction suggests that the fish tends to look to some specific directions. From the diagram it is observed that the horizontal body direction appears seldom, tilting always upward or downward. From the velocity diagram it is also observed that the fish moves on along the vessel wall almost horizontally and/or vertically. As for acceleration, it is similar to the velocity charateristics. Another comprehensive presentation is the use of spherical or polar diagram. Figure 11 shows such directions of velocity and acceleration. A direction form the origin to each point on the sphere shows the corresponding direction of the movement or acceleration. Above mentioned tendencies are intuitively shown in the figure. The polar diagram serves comprehensive understanding of directions of the body as well (data not shown).

Velocity, acceleration and energy associated with the swimming in the consecutive 21 time intervals, in 100 seconds, are shown in Fig. 12. Kinetic energy during the swimming is calculated from the square of the magnitude of the velocity.

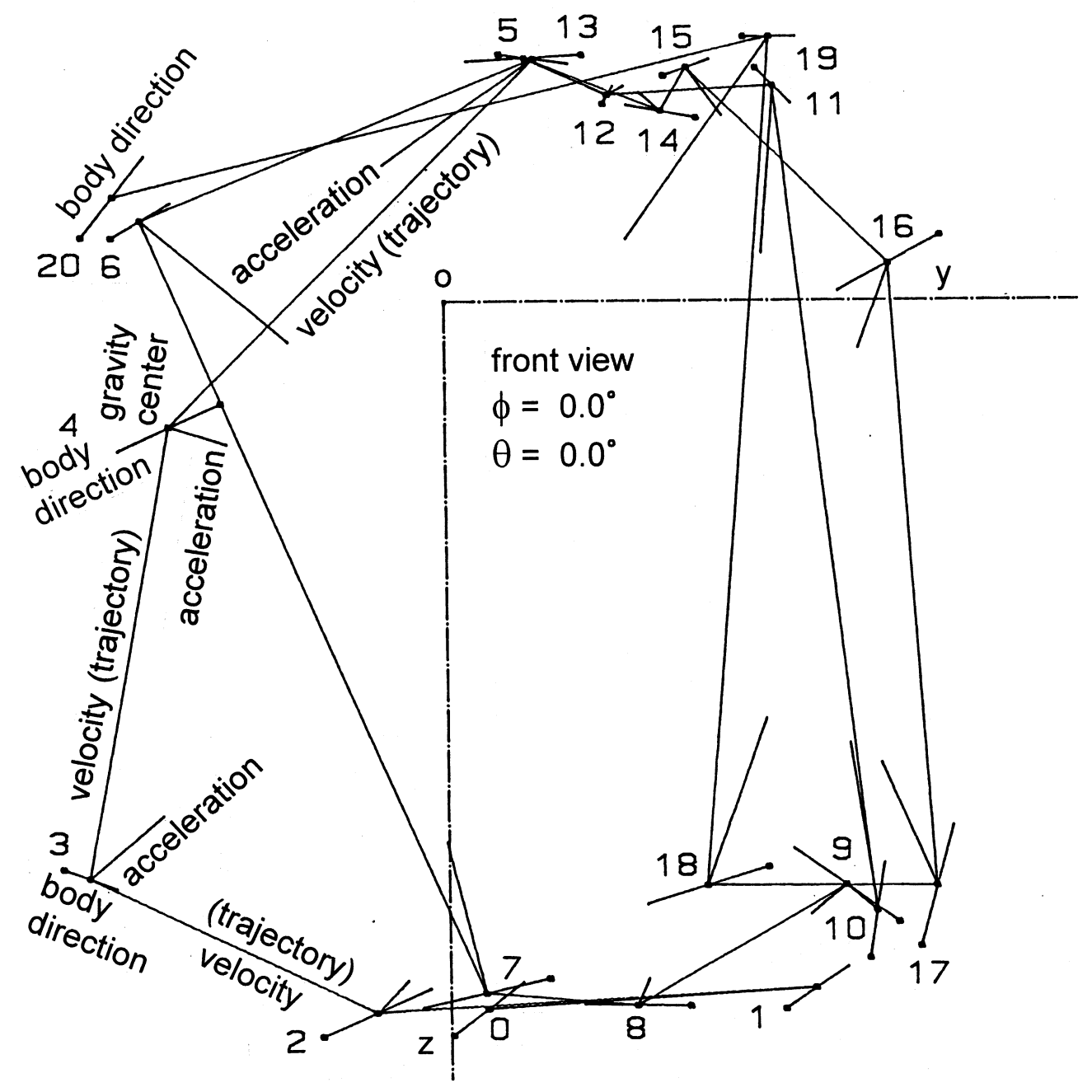

Fig. 9. Front view of the fish behavior with tangential body direction. 
From these three diagrams it is observed that the fish moves intermittently, taking some ten seconds' recess. It seems that the fish lacks the constitutional power necessary for continuous swimming.
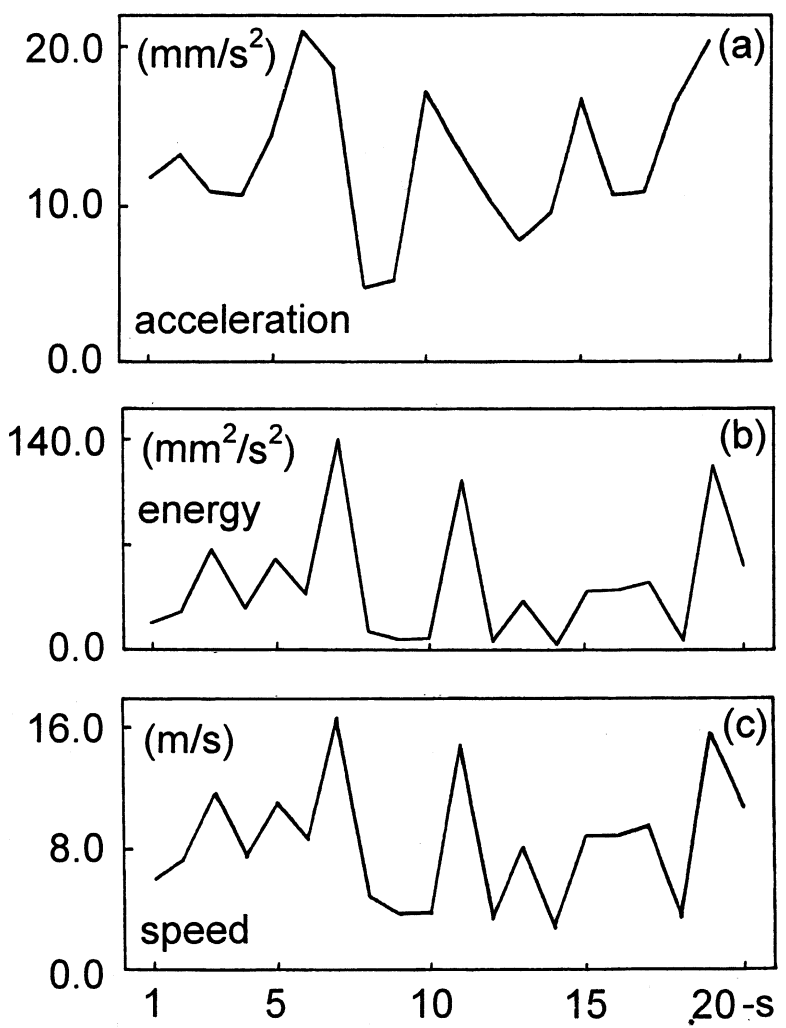

Fig. 12. Speed, acceleration and energy during elapsed time of swimming.

\section{Concluding Remarks}

Behavioral feature extraction for a small swimming fish in a water vessel is made. It is based on the usage of sequentially generated threedimensional geometric models that are reconstructed from the three-directional video images. With these models, quantitative analysis of time-to-time varying characteristics of the behavior is demonstrated. This three-dimensional tracking is more informative, compared with the conventional two-dimensional image handling. It gives three-dimensional and quantitative information concerning the twisted body deformation, positions, directions, angles and kinetic parameters relevant to behavioral factors. Physical strength will be estimated by this method. What the fish is doing can even be estimated by the time sequential shape and motion analysis. Three-dimensional computer graphics will be informative and suitable for this application.

In this visualization a single object is dealt with, however, the principal procedure is the same for multiple objects if the number is not so large. The modeling needs neither specific light source nor specific light pattern projection to the object. From the image acquisition for the threedimensional shape approximation to the feature extraction for the behavior analysis, the process is carried out automatically without manual operation.

The output format of this modeling is compatible with the boundary data formats that are used in many computer codes for the flow and combustion simulation, solid mechanics, and CAD applications. Swimming mechanism of the fish will be investigated by combining with the numerical fluid dynamics, where boundary mesh data are automatically generated using this modeling. This development will have some considerable utility in the integration of behavior analysis by experiment with those carried out by kinetic modeling and numerical simulation.

\section{References}

[1] J. DOI, T. MiYAKE, T. ASANUMA, Three-dimensional flow analysis by on-line particle tracking. In Flow Visualization III, (W. J. Yang Ed.), (1987) pp. 229-234. Hemisphere-Springer.

[2] J. DOI, K. FUJIMATA, T. MiYAKE, Three-dimensional boundary element analysis with automated solid modeling system, In Advances in Boundary Elements (C. A. Brebbia, J. J. ConnorEds.), (1990), Vol. 3 (Stress Analysis), pp. 405-416. Computational Mechanics-Springer.

[3] J. DOI, T. MIYAKE, Automated geometric modelling of a tillage blade. Journal of Agricultural Engineering Research, 55 (1993), pp. 207-216.

[4] M. MASUYA, J. DOI, Extraction of three-dimensional multiple skeletons and digital medial skeletons, $I E$ ICE (Institute of Electronics, Information and Communication Engineers) Trans. on Information and Systems, E-78D (1995), pp. 1567-1572.

Received: October, 2000 Accepted: November, 2000 


\author{
Contact address: \\ Junta Doi, Professor \\ Department of Computer Science \\ Chiba Institute of Technology \\ 2-17-1 Tsudanuma \\ Narashino, 275-0016 \\ Japan \\ e-mail: doi@cs.it-chiba.ac.jp \\ Tel: +81-47-478-0546 \\ Fax: +81-47-478-0549
}

JUNTA DOI is a professor emeritus of the University of Tokyo, where he established the laboratory of bioinformation engineering and the department of biotechnology. He is currently a professor in the department of computer science at Chiba Institute of Technology. His research interests include bioinformatics, molecular modeling and image processing. $\mathrm{He}$ is a member of the IEEE and the IEEE Computer and Engineering in Medicine and Biology Societies.

TETSUO MIYAKE is currently an associate professor in the department of production systems engineering at Toyohashi University of Technology, before which he worked at the Central Research Laboratory of Asahi Glass Company. His research interests include non-contact measurement, motion tracking and human interface. He received a $\mathrm{PhD}$ in electrical and electronic engineering from the University of Tokyo. $\mathrm{He}$ is a member of the IEEE Computer Society. 

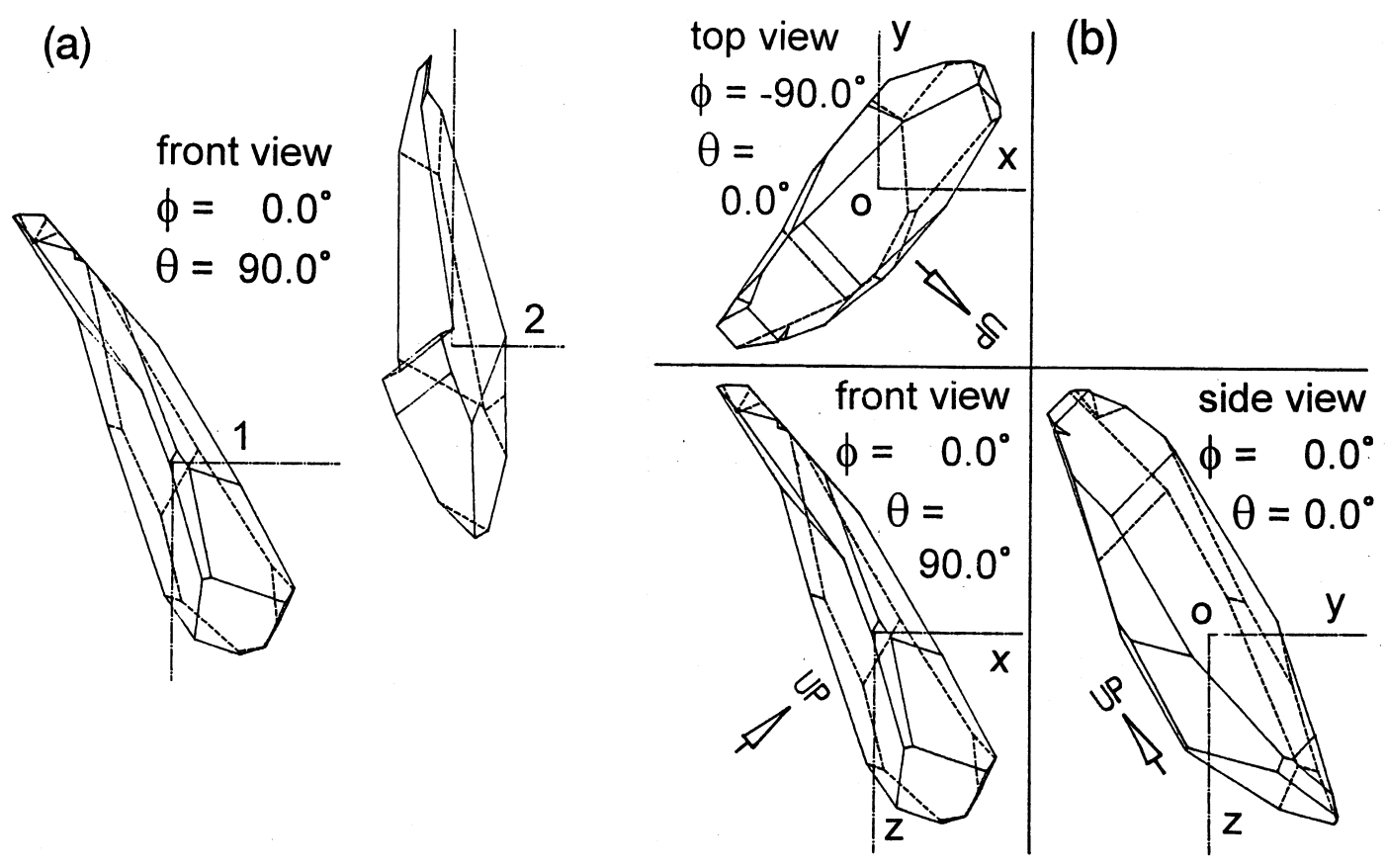

Fig. 5. Three-directional modeling of the fish at two moments.

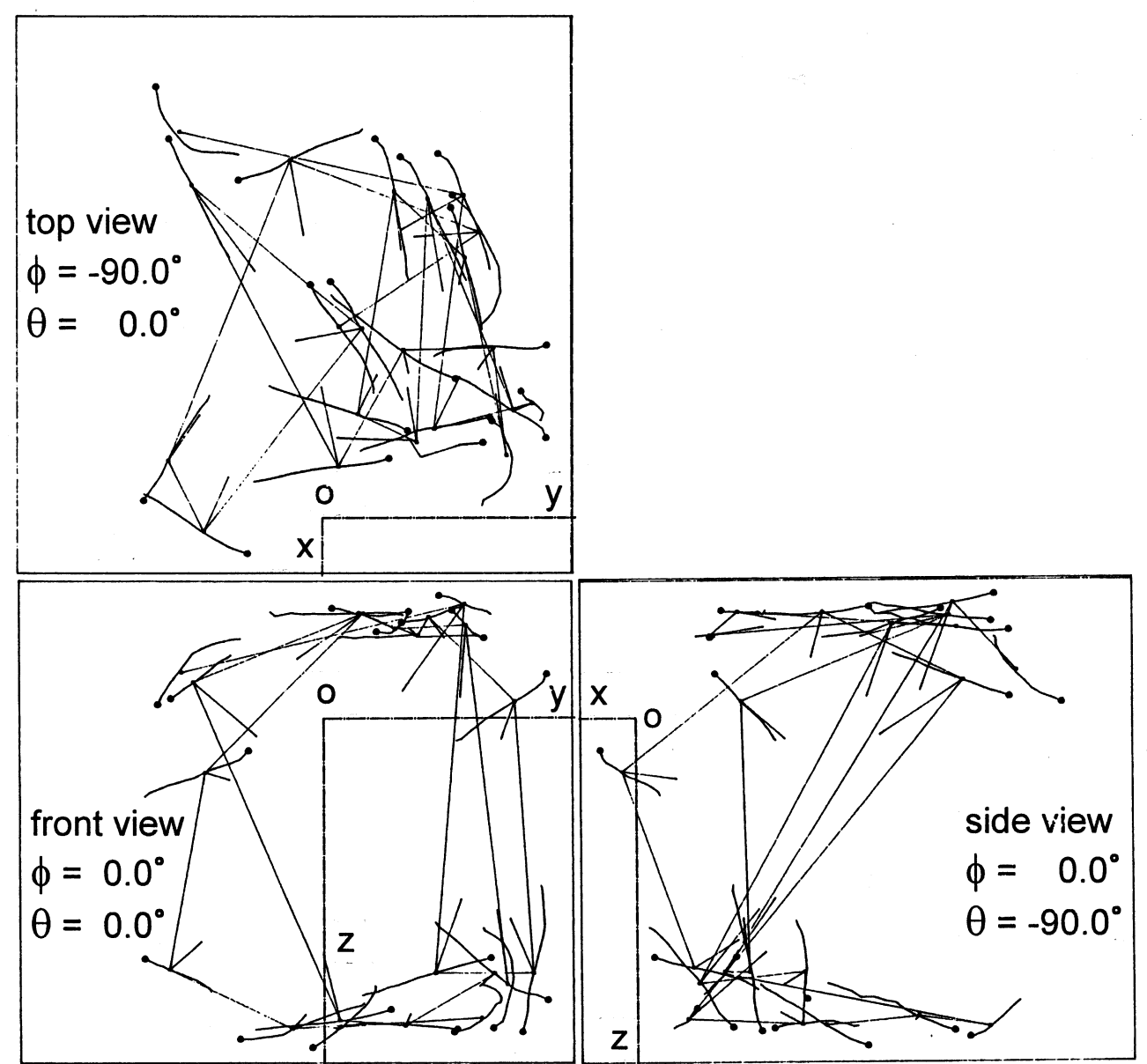

Fig. 6. Third angle projection of the fish behavior. Each curved line segment with a dot at the one end is its skeleton. The dotted end point is the mouth and the opposite end is the tail. A cross point in this skeleton is the gravity center, which represents the body position. 


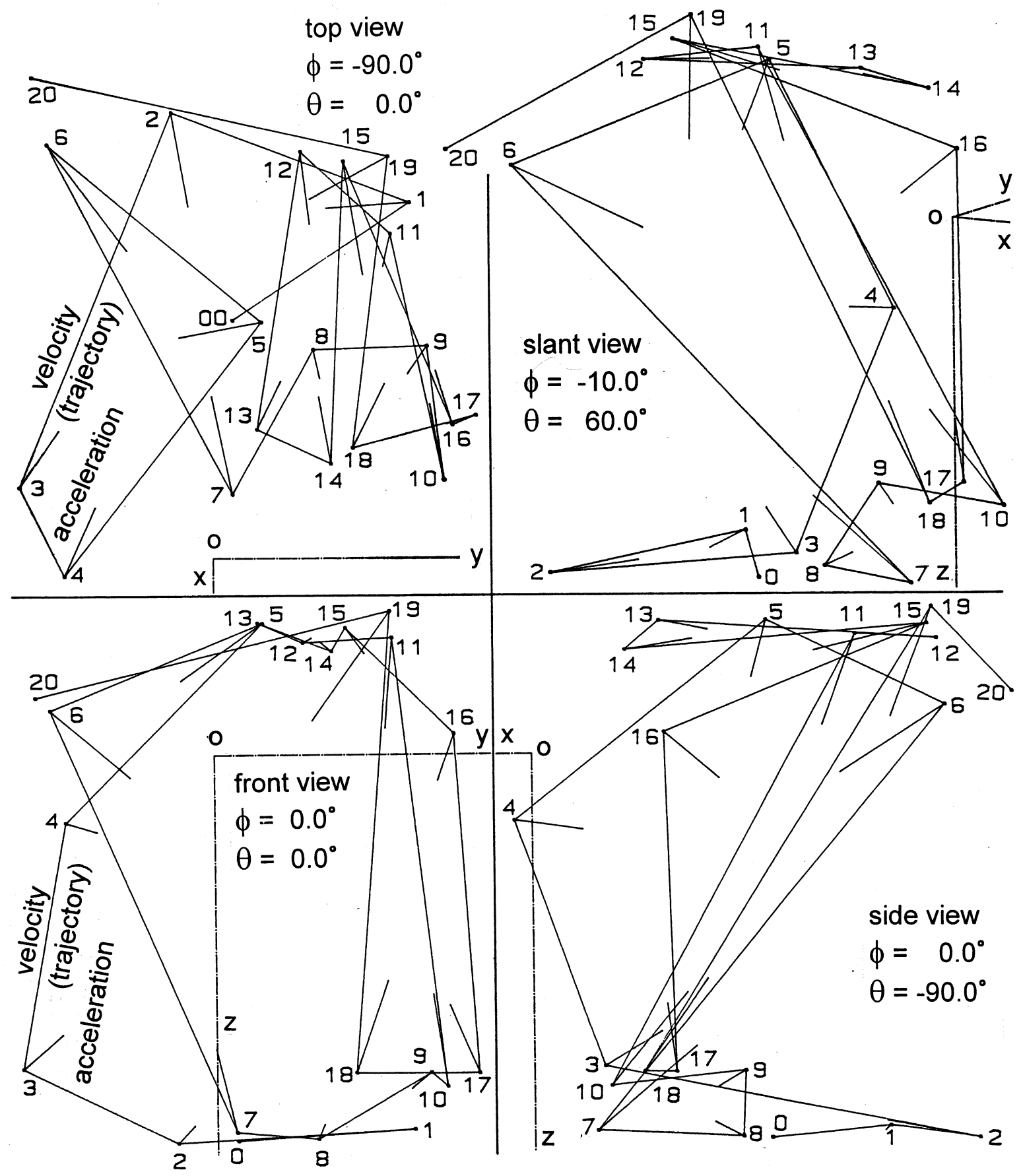

Fig. 8. Third angle projection of the fish behavior with the fourth angle projection. 


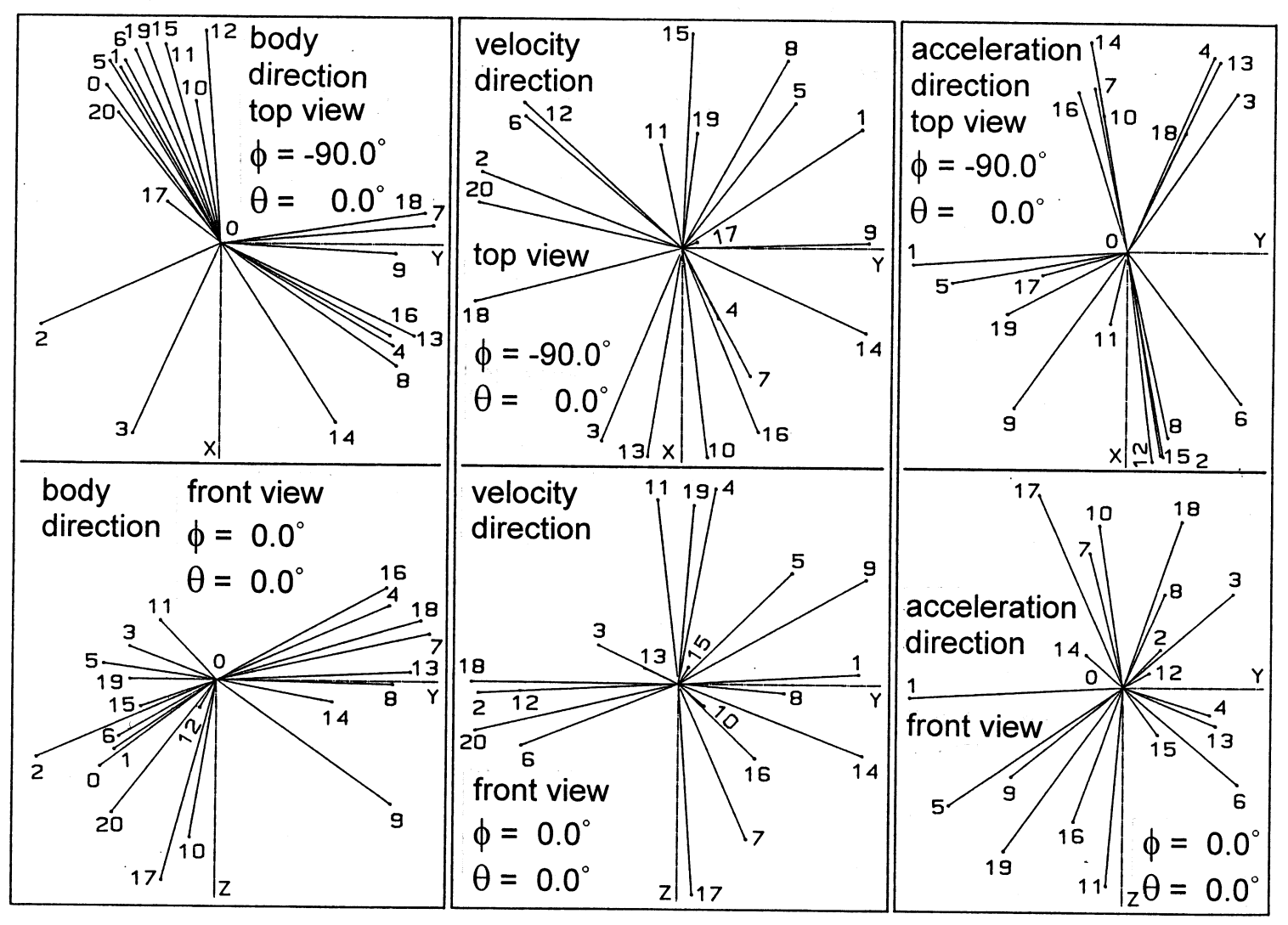

Fig. 10. Directions of the fish body, velocity and acceleration.

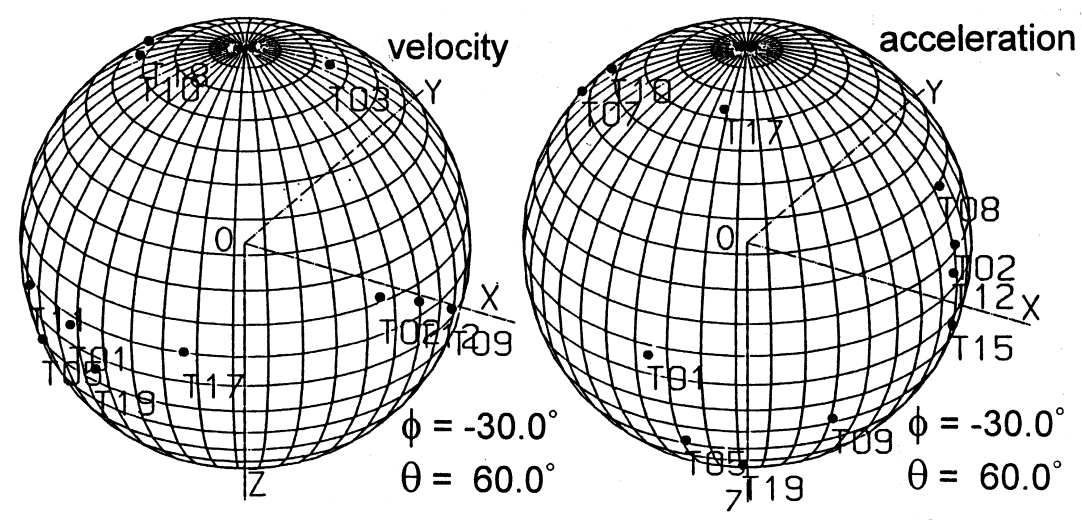

Fig. 11. Spherical coordinate representation of directions of velocity and acceleration. Hidden points are suppressed. 